\title{
Better stress coping associated with lower tau in amyloid-positive cognitively unimpaired older adults
}

Eider M. Arenaza-Urquijo, PhD, Scott A. Przybelski, BS, Mary M. Machulda, PhD, David S. Knopman, MD, Val J. Lowe, MD, Michelle M. Mielke, PhD, Ashritha L. Reddy, MS, Yonas E. Geda, MD, Clifford R. Jack, Jr., MD, Ronald C. Petersen, MD, PhD, and Prashanthi Vemuri, PhD

Neurology ${ }^{\circledR}$ 2020;94:e1571-1579. doi:10.1212/WNL.0000000000008979

\author{
Correspondence \\ Dr. Vemuri \\ Vemuri.prashanthi@ \\ mayo.edu
}

\begin{abstract}
Objective

Research in animals has shown that chronic stress exacerbates tau pathology. In humans, psychological stress has been associated with higher risk of Alzheimer disease clinical syndrome. The objective of this cross-sectional study was to assess the hypothesis that stress coping ability (assessed via the Brief Resilience Scale $[\mathrm{BRS}]$ ) is associated with tau burden and to evaluate whether these associations differed by sex and amyloid status $(\mathrm{A}+/ \mathrm{A}-)$ in cognitively unimpaired (CU) older adults.
\end{abstract}

\section{Methods}

We included $225 \mathrm{CU}$ participants (mean age $70.4 \pm 10.2$ years, $48 \%$ female) enrolled in the population-based Mayo Clinic Study of Aging who completed the BRS and underwent amyloid-PET (Pittsburgh compound B-PET) and tau-PET (AV1451-PET). We fitted multiple regression and analysis of covariance models to assess the associations between BRS and tauPET and the interaction with amyloid status and sex. We focused on entorhinal cortex (ERC) tau burden and also performed voxel-wise analyses. Age, sex, education, depression, and anxiety were considered as covariates.

\section{Results}

Higher stress coping ability was associated with lower tau burden in the medial temporal lobe (including ERC) and occipito-temporal and cuneal/precuneal cortices. The association was present in both $\mathrm{A}+$ and $\mathrm{A}-$ but weaker in $\mathrm{A}-\mathrm{CU}$ older adults. There was an interaction between amyloid status and stress coping ability that was restricted to the medial temporal lobe tau such that A+ CU older adults with lower stress coping abilities showed higher tau. There were no significant interactions between stress coping and sex.

\section{Conclusions}

A faster termination of the stress response (higher coping ability) may limit the negative effects of stress on tau deposition. Conversely, lower stress coping ability may be an early sign of accumulating tau pathology. Longitudinal studies are warranted to clarify whether stress mechanisms act to exacerbate tau pathology or tau influences stress-related brain mechanisms and lowers the ability to cope with stress. 


\section{Glossary}

$\mathrm{AD}=$ Alzheimer disease; ANCOVA = analysis of covariance; $\mathbf{B R S}=$ brief resilience scale; $\mathbf{C U}=$ cognitively unimpaired; $\mathrm{ERC}=$ entorhinal cortex; HPA = hypothalamus-pituitary-adrenal cortex; $\mathbf{M C I}=$ mild cognitive impairment; $\mathbf{M C S A}=$ Mayo Clinic Study of Aging; ROI = region of interest; SUVR = standard uptake value ratio.

Identifying modifiable factors associated with resistance to Alzheimer disease $(\mathrm{AD})$ pathologies-amyloid and tau-is a fundamental step towards designing successful interventions and ultimately towards delaying the onset of $\mathrm{AD}$ clinical syndrome. ${ }^{1,2}$

Several studies have assessed associations of in vivo amyloid measurements and modifiable factors in cognitively unimpaired individuals. Sleep disruption, ${ }^{3,4}$ physical activity levels, ${ }^{5-7}$ and intellectual enrichment ${ }^{8-12}$ have all been associated with amyloidosis, although some of these associations remain controversial. ${ }^{11}$ While there is increasing interest in the therapeutic potential of targeting tau, ${ }^{13,14}$ studies assessing these types of associations with in vivo tau measurements are sparse. $^{15,16}$

In the present study, we hypothesized chronic stress as one of the potential factors associated with tau deposition. The major neuroendocrine response to stress is via activation of the hypothalamus-pituitary-adrenal cortex (HPA) axis that leads to glucocorticoid secretion. ${ }^{17,18}$ Research in animals suggests that stress responses-through the mediation of glucocorticoids-may trigger tau-dependent mechanisms and lead to a greater sensitivity to the deleterious action of amyloid. ${ }^{19-22}$

In humans, chronic stress is associated with increased risk of $\mathrm{AD}$ clinical syndrome $\mathrm{e}^{23-27}$ and high glucocorticoid levels are associated with accelerated brain aging and cognitive decline. ${ }^{28-32}$ Further, patients with mild cognitive impairment (MCI) and patients with $\mathrm{AD}$ show higher glucocorticoids levels than controls. ${ }^{31,33,34,}$ In cognitively unimpaired (CU) participants, high cortisol levels may exacerbate the effects of amyloid on cognitive decline. ${ }^{35}$ These studies support a role of stress in exacerbating aging and disease, but the link between chronic stress and $\mathrm{AD}$ pathology has not been established in humans. ${ }^{36}$

There is significant variability in stress responses that ranges from depression ${ }^{37,38}$ to stress resilience, which is defined by the ability to cope well with stressors or to recover after stressor exposure. ${ }^{39,40}$ Older adults with high levels of stress resilience-or stress coping ability-require less time to recover and terminate the stress response after experiencing stressor exposure, which is essential for reducing the damaging effects of chronic elevations of glucocorticoids. ${ }^{41}$ Importantly, while stress coping ability can be shaped by individual factors such as age and sex, this ability can be enhanced by training ${ }^{42}$ and uniquely contributes to health outcomes later in life, ${ }^{43}$ making it an important target to evaluate for interventions.

Building on the evidence from animal and human research, we hypothesized that better stress coping strategies would be associated with resistance to tau. The specific goals of the study were to (1) examine the cross-sectional association of in vivo measurements of tau burden as measured by tau-PET with stress coping ability in CU older adults and (2) assess the interaction among amyloid status (A+/A-), sex, and stress coping ability on tau burden. We performed regional analyses focused on the entorhinal cortex (ERC), a region showing early tau deposition and highly vulnerable in aging and dementia, ${ }^{44}$ and voxel level associations to further explore the topography of the association.

\section{Methods}

\section{Participants}

Participants were selected from the Mayo Clinic Study of Aging (MCSA), a population-based study started in 2004 among Olmsted County, Minnesota, residents aged 50-89 years. The Olmsted County population was enumerated using the Rochester Epidemiology Project medical records linkage system. ${ }^{45,46}$ Details about study design and clinical diagnostic criteria are discussed elsewhere. ${ }^{46,47}$ For the present study, we included $225 \mathrm{CU}$ participants aged 50 years and older who had amyloid-PET, tau-PET, and stress coping ability assessments available (see below). Participants completed the assessments between June 2015 and June 2018. The mean time delay between the tau scan and the stress coping ability assessment was $1.4 \pm 0.88$ years. The results presented in this study are not adjusted by the time delay between assessments, but the relationships between our variables of interest did not differ when the time delay was introduced as covariate in the statistical models.

\section{Standard protocol approvals, registrations, and patient consents}

The institutional review boards of both Mayo Clinic and Olmsted Medical Center approved this study. All participants provided written informed consent.

\section{Stress coping ability assessment}

Stress coping ability or resilience to stress was assessed using the brief resilience scale (BRS). The BRS includes 6 items that are framed with regard to negative events. Higher scores indicate more resilience. In contrast to other measurements focusing on resilience resources, this scale assesses the ability to recover from stress itself (e-Methods 1, doi.org/10.5061/dryad.0zpc866t7). ${ }^{43}$ 
Depressive and anxiety symptoms were measured using the Beck Depression Inventory-II and Beck Anxiety Inventory. ${ }^{48,49}$

\section{Imaging analyses and preprocessing}

\section{Amyloid- and tau-PET scans}

Amyloid-PET and tau-PET images were acquired with PET-CT operating in $3 \mathrm{D}$ mode. The details of the acquisition and processing were published previously. ${ }^{50}$ In brief, an in-house modified version of the automated anatomic labeling atlas was used and the atlas-based parcellation of the PET images into regions of interest was done in the participant's native anatomical space.

\section{Amyloid deposition}

Global cortical amyloid-PET retention ratio was computed as previously reported: the median uptake of voxels in the prefrontal, orbitofrontal, parietal, temporal, anterior cingulate, and posterior cingulate/precuneus regions of interest (ROIs) for each participant were divided by the median uptake over voxels in the cerebellar crus ROI of the atlas. ${ }^{\text {el }}$ We used a global standard uptake value ratio (SUVR) cutoff of 1.48 to determine amyloid abnormality or positivity, which was established using the methodology we published previously but with updated processing pipelines. ${ }^{50}$

\section{Tau deposition}

Tau-PET uptake was divided by the median uptake in the cerebellar crus to create standard uptake value ratios (SUVRs). ${ }^{\text {2 }}$ Entorhinal tau-PET was assessed by averaging the uptake in the left and right ERC. For voxel-wise analyses, we used tau-PET images normalized to the Mayo Clinic Adult Lifespan Template ${ }^{\mathrm{e} 3}$ and smoothed using a 6-mm full-width at half maximum Gaussian kernel. For the main analyses, including the voxel-wise analyses, we did not partial volume correct the tau-PET signal, but the main results presented here did not differ with partial volume correction.

\section{Statistical analyses}

The characteristics of the sample are described using mean and SD for continuous variables and count and percentage for categorical variables. The associations between stress coping ability and demographic variables were assessed using Pearson correlations and $t$ tests.

ROI analyses were performed in IBM SPSS Statistics version 20.0 (SPSS Inc., Chicago, IL) and voxel-wise analyses were performed in SPM12.

Statistical analyses were carried out first to test the association of BRS with tau region-wise and voxel-wise and second to test the interaction with age and sex, region-wise and voxel-wise.

\section{Association between BRS and tau burden}

\section{Regional analyses}

We tested the association between ERC tau and BRS scores using an analysis of covariance (ANCOVA) model, with age, sex, and education as covariates.

\section{Voxel-wise analyses}

We ran voxel-wise multiple regression analyses in SPM12 including tau-PET scans as the dependent variable and BRS scores as the independent variable with age, sex, and education as covariates.

In both regional and voxel-wise analyses we also examined models adjusted by depression and anxiety scores.

\section{Interaction among BRS, amyloid status, and sex}

\section{Regional analyses}

We included amyloid status as a factor in the ANCOVA model described above and tested for BRS-by-amyloid and BRS-by-sex interactions.

\section{Voxel-wise analyses}

To test for BRS-by-amyloid and BRS-by-sex interactions, we fitted separate one-way ANCOVA models in SPM12. The first model included amyloid status as a factor, tau-PET scans as dependent variables, BRS as independent variables, and age, sex, and education as covariates. The second model included sex as a factor, tau-PET scans as dependent variables, and BRS as independent variable, and was adjusted by age and education.

We restricted the voxel-wise analyses to the gray matter using an explicit mask that included voxels with a gray matter probability greater than 0.2 . Voxel-wise results were considered significant at uncorrected $p<0.005$ at voxel level and family-wise error corrected $p<0.05$ at cluster level. Taking into account the expected effect, the number of covariates, and the sample size, we applied a more liberal threshold of uncorrected $p<0.005$ at voxel level and a $\mathrm{K}>500 \mathrm{~mm}^{3}(150$ voxels) when testing voxel-wise interactions.

\section{Data availability}

Data that support the findings of this study are available upon reasonable request from the Mayo Clinic Study of Aging investigators.

\section{Results}

\section{Sample characteristics}

Demographic characteristics of the study sample are provided in the table. Stress-coping was not associated with age $(r=$ $-0.09 ; p=0.20)$, amyloid status (amyloid positivity) $(p=0.27)$, or sex $(p=0.99)$. Higher BRS was associated with higher education $(r=0.24 ; p<0.001)$. There were moderate associations between higher BRS and lower scores in depression $(r=$ $0.4 ; p<0.001)$ and anxiety scales $(r=-0.3 ; p<0.001)$.

\section{Association between BRS and tau burden}

\section{Regional analyses with ERC tau}

Results from the multiple regression model to predict ERC tau showed a significant effect of BRS $(F=13.89, p<0.001)$ adjusted by age, sex, and education. Further adjustments by 
Table Demographic characteristics of the study sample $(n=225)$

\begin{tabular}{lll}
\hline & Mean (SD) or $\mathbf{n}(\%)$ & Range \\
\hline Age, $\mathbf{y}$ & $70.44(10.20)$ & $52-98$ \\
\hline Male sex & $117(52)$ & $7-20$ \\
\hline Education, y & $14.96(2.47)$ & $23-30$ \\
\hline MMSE & $28.74(1.14)$ & $1.33-5$ \\
\hline Stress coping scale & $3.80(0.68)$ & $0-23$ \\
\hline Beck Anxiety Inventory & $2.20(3.80)$ & $0-22$ \\
\hline Beck Depression Inventory & $4.29(4.35)$ & - \\
\hline Pittsburgh compound B-positive & $78(35)$ & - \\
\hline APOE4 & $65(30)$ & \\
\hline
\end{tabular}

Abbreviation: MMSE = Mini-Mental State Examination.

anxiety and depression scores did not alter the results $(F=9.7$, $p=0.002)$. Neither anxiety nor depression scores were significant contributors to the model (anxiety: $F=0.30, p=0.60$; depression: $F=0.80, p=0.37$ ).

\section{Voxel-wise associations between stress coping ability and tau}

Higher BRS scores were associated with lower tau deposition in the inferior temporal gyrus, ERC, parahippocampus, hippocampus, lingual gyrus, fusiform cortex, occipital pole, and cuneal and precuneus cortices after adjusting for age, sex, and years of education (figure 1). The topographic pattern was unchanged with partial volume correction (figure e-1, doi. org/10.5061/dryad.0zpc866t7) and when adjusting for depression and anxiety (figure e-2, doi.org/10.5061/dryad. 0zpc866t7). In a sensitivity analysis, we found that BRS was not associated with neurodegeneration in $\mathrm{AD}$ regions $(F=$ $0.78, p=0.38$; adjusted by sex, age, and education), further supporting the tau associations with BRS.

We assessed the association separately in A+ and A- subgroups. While the association was present in both subgroups, the effect was weak in $\mathrm{A}-$ and moderate in $\mathrm{A}+$ (figure 2).

Overall, results from regional and voxel-wise analyses show a main effect of BRS on tau burden including, but not restricted to, areas of early tau accumulation.

\section{Interaction among BRS, amyloid status, and sex}

\section{Regional analyses on ERC tau}

There was a significant BRS-by-amyloid status interaction $(F=6.98, p<0.009)$. When tested separately in A+ and Asubgroups, the association was reduced to a trend in A- older adults $(F=2.93, p=0.089)$ but was still significant in the A+ group $(F=11.16, p=0.001)$. To assess the specificity of the interaction to this region, we repeated the model in the inferior temporal lobe, a region of early tau deposition but outside the medial temporal lobe. There was no significant interaction between BRS and amyloid on inferior temporal lobe tau $(F=1.50, p=0.22)$. The results of these 2 areas are contrasted in figure 3. Finally, there was no significant interaction between sex and BRS on ERC tau $(F=0.5, p=0.82)$.

Further adjustments by anxiety and depression scores did not alter the significance of the results (BRS-by-amyloid: $F=6.6$, $p<0.01$; BRS-by-sex: $F=0.01, p=0.91)$. Neither anxiety nor depression scores were significant contributors to the model (anxiety: $F=0.14, p=0.71$; depression: $F=0.72, p=0.40$ ).

\section{Voxel-wise analyses}

We found a BRS-by-amyloid status interaction that was restricted to medial temporal regions including mainly the bilateral parahippocampal cortex and amygdala (left greater than right) and the left ERC, and to a lesser extent, the bilateral anterior hippocampus (figure 3 ). The pattern of results was similar when we further controlled for anxiety and depression scores (figure e-3, doi.org/10.5061/dryad. 0zpc866t7), and there were no significant effects of anxiety or depression scores on tau. There was no significant BRS-by-sex interaction.

Overall, results from regional and voxel-wise analyses show an interaction of BRS and amyloid status restricted to the medial temporal lobe and notably the ERC. The results were unchanged when we repeated the analyses without the participant showing the lowest BRS scores and higher tau-PET uptake.

\section{Discussion}

The availability of in vivo measurements of amyloid and tau has increased interest in identifying modifiable factors that may play a role in the development and promotion of $\mathrm{AD}$ 
Figure 1 Results from the voxel-wise multiple regression analysis between stress coping ability and tau-PET adjusted by sex, age, and education

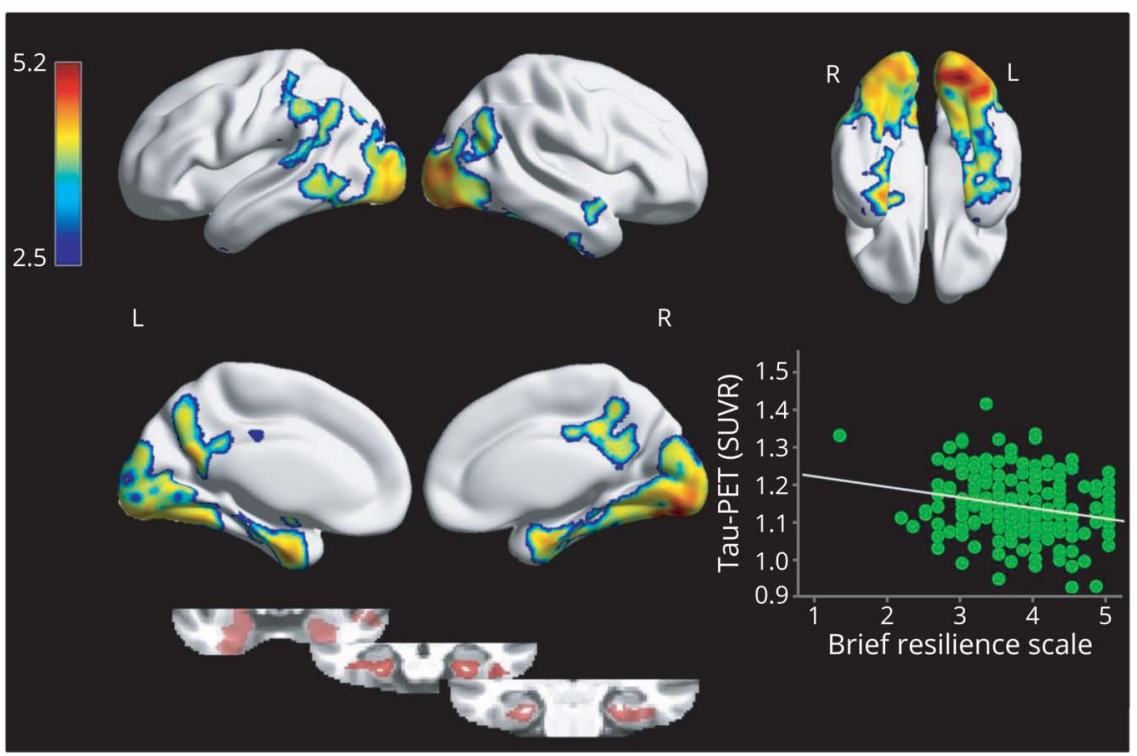

Maps were thresholded at $p<0.005$ at voxel level and family-wise error $p<0.05$ at cluster level. Significant clusters included inferior and medial temporal lobes, occipito-temporal (fusiform and lingual gyri), and cuneal/precuneus cortices. The $Y$ axis represents the mean standard uptake value ratio (SUVR) tau-PET uptake within these regions. The $X$ axis represents the brief resilience scale scores. Medial temporal results are shown as a binary mask overlaid into a T1 structural image. pathologies. In this study, we investigated stress coping ability-or resilience to stress - in relation to tau deposition in cognitively unimpaired older adults. The main findings were (1) better stress coping ability was associated with lower tau deposition in the inferior and medial temporal lobes, occipito-temporal (fusiform and lingual gyri), and cuneal/ precuneus cortices; (2) although this association was present in both $\mathrm{A}+$ and $\mathrm{A}$ - participants, the effect was moderate in the full sample, stronger among $\mathrm{A}+$ and weaker among $\mathrm{A}$ - older adults; (3) amyloid status interacted with stress coping ability on regions showing early tau accumulation, that is, on medial temporal regions including the ERC, such that A+ participants with lower stress coping abilities were associated with higher tau.

Figure 2 Plots show the association between mean tau-PET uptake of the areas from the voxel-wise analysis (inferior and medial temporal lobes, occipito-temporal and cuneal/precuneus cortices, $Y$ axis) and stress coping scale in amyloid-positive and amyloid-negative participants ( $X$ axis)
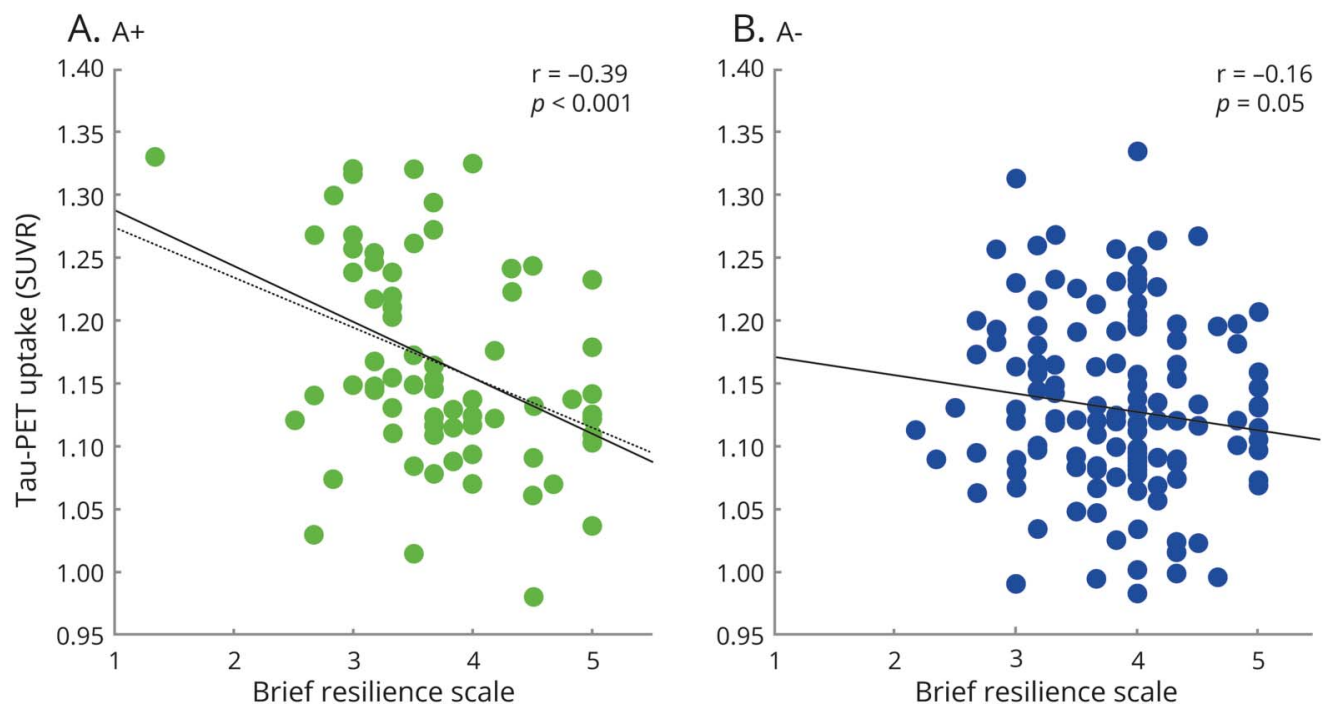

(A) Amyloid-positive group. (B). Amyloid-negative group. The discontinuous line in the $A+$ group plot (A) represents the regression line without the participant showing the lowest stress coping score $(r=-0.36 ; p=0.002)$. SUVR = standard uptake value ratio. 
Figure 3 Results from the regional analyses showing an interaction between stress coping scale and amyloid status on entorhinal tau standard uptake value ratios (SUVRs) but not on inferior temporal tau SUVRs and results from the voxel-wise analyses showing an interaction between stress coping ability and amyloid status

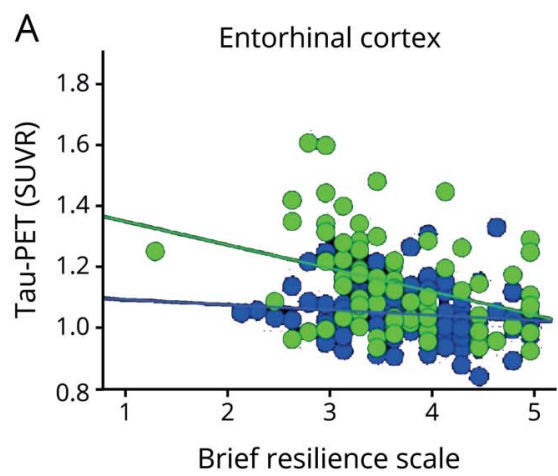

Brief resilience scale
- A+ - A-

Inferior temporal gyrus

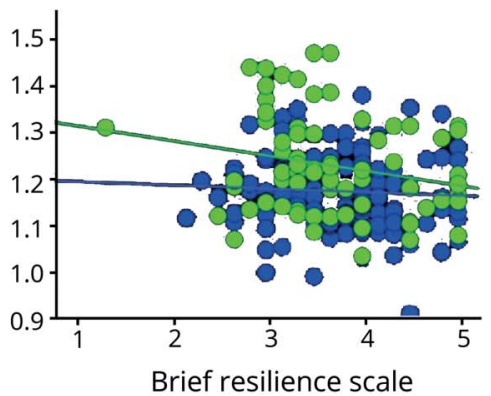

(A) Results from the regional analyses show an interaction between stress coping scale and amyloid status on entorhinal tau SUVRs (left) but not on inferior temporal tau SUVRs (right). (B) Results from the voxel-wise analyses show an interaction between stress coping ability and amyloid status. Maps were thresholded at $p<0.005$ at voxel level. Medial temporal results are shown as a binary mask overlaid on a T1 structural image. Both analyses were adjusted for age, sex, and years of education.
To our knowledge, this is the first study to investigate associations between stress responses and in vivo tau measurements using flortaucipir PET. We found that greater stress coping ability was associated with lower tau. The results show a specific association of tau with stress - and not with amyloid or neurodegeneration - thus suggesting an early mechanistic link between tau and stress. This association was not restricted to areas of early tau deposition but included the inferior and medial temporal lobes, occipito-temporal (fusiform and lingual gyri), and cuneal/precuneus cortices. A previous study in MCSA participants suggested that tau-PET signal is not confined to medial temporal regions in cognitively unimpaired individuals $(\mathrm{A}+$ or $\mathrm{A}-){ }^{\mathrm{e} 4}$

The present study was cross-sectional and, as a result, causal relationships cannot be established. Further, the association between stress coping ability and the biological stress response is speculative. Our results can be interpreted using 2 distinct arguments as discussed further below: (1) a maintained stress response (lower ability to cope or recover from stress) may trigger or exacerbate tau pathology; or (2) lower stress coping ability may be an early sign of accumulating tau pathology.

Studies in mice have reported associations between elevated glucocorticoid levels and increased tau accumulation suggesting a modulation of glucocorticoids on tau deposition. ${ }^{19-21}$ In this study, stress coping ability was measured as the ability to bounce back and recover from stress. ${ }^{43}$ This assessment likely captures more of a stable personality trait. Higher stress coping ability implies a faster recovery after stressor exposure and thus may imply a faster termination of the stress response, which involves the HPA axis and leads to glucocorticoid secretion. ${ }^{17,18}$ Thus, a tentative mechanistic explanation for our results is that a faster termination of the stress response may limit the negative effects that elevated levels of glucocorticoids may have on tau deposition. Measuring glucocorticoids in the future may further help clarify 
the causal mechanisms through which stress response and tau deposition are related.

Stress coping strategies did not differ by amyloid status. This suggests that amyloid did not disrupt the ability to cope with stress. However, the association between stress and tau was stronger in $\mathrm{A}+$ than $\mathrm{A}-\mathrm{CU}$ older adults. A+ CU older adults with lower stress coping ability had increased medial temporal tau, mainly the ERC and parahippocampal cortex, but not in other areas of early accumulation. This result could be explained by the fact that $\mathrm{A}+$ have a wider range of tau values to detect these associations. Previous studies in animals suggest that stress may play a role in the development and progression of $\mathrm{AD}$ pathologies $20,21, \mathrm{e} 6-8$ with tau hyperphosphorylation mediating the effects of stress on neurodegeneration ${ }^{22}$ and cognition ${ }^{19}$ even in the absence of amyloid. The present results thus suggest the hypothesis that stress may play a double role as a trigger of tau in aging independently of amyloid and as an accelerator of the biomarker cascade in older adults with $\mathrm{AD}$ pathologic change, promoting medial temporal lobe tau in the presence of amyloid. Previous studies showed that high cortisol levels were associated with accelerated cognitive decline in cognitively impaired-but not unimpaired-participants ${ }^{30,31}$ and suggested that cortisol dysregulation modulates the downstream clinical expression of $\mathrm{AD}$ pathology. ${ }^{33}$ High cortisol levels may also accelerate the effect of amyloid positivity on cognitive decline in cognitively unimpaired participants. ${ }^{35}$ Further studies are needed to understand the role that tau pathology plays on the described effects.

The topography of the association between stress coping ability and tau deposition included brain regions involved in the stress response such as the amygdala. ${ }^{\mathrm{e} 9}$ Thus, it is possible that tau deposition influences stress-related brain mechanisms and lowers the ability to cope with stress. ${ }^{\text {e10 }}$ This argument is supported by the literature showing HPA axis dysregulation in $\mathrm{MCI}$ and $\mathrm{AD} .^{31,33,34}$ Further, neuropsychiatric symptoms are present early in $\mathrm{AD},{ }^{\mathrm{e} 11}$ and are associated with faster cognitive decline. ${ }^{\mathrm{e} 2}$ Indeed, previous studies showed that prolonged stress exposure was associated with depression, ${ }^{37,38}$ which often co-occurs with anxiety, both associated with increased risk of $\mathrm{AD}$ clinical syndrome. ${ }^{\mathrm{e} 3, \mathrm{e} 14}$ The cognitive debt hypothesis ${ }^{\mathrm{e} 15}$ proposes a framework where a cognitive process-repetitive negative thinking-may activate the stress response and underlie the risk associated with several neuropsychiatric symptoms. In line with this, the present findings add to recent preliminary evidence linking depression to in vivo tau measurements. ${ }^{\mathrm{e}}{ }^{16}$ The association between stress coping and tau, however, remained significant after adjusting for depression and anxiety scores, and thus low stress coping ability may represent an earlier manifestation or have an independent role.

In the present study, we did not find a significant association between stress-coping ability and neurodegeneration in $\mathrm{AD}$ regions. This result suggests that the association between tau and stress precedes neurodegeneration in these areas. In previous studies, tau deposition in the ERC has been associated with episodic memory and subjective cognitive decline independent of amyloid. ${ }^{\mathrm{e} 7, \mathrm{e} 18}$ An important avenue of future research therefore will be to understand the role that stress may play in primary age-related tauopathy. ${ }^{\mathrm{e} 9}$

A recent study in $\mathrm{CU}$ women reported an association between midlife stress and total CSF tau suggesting an association between stress and nonspecific neurodegenerative processes but not the core biomarkers of $\mathrm{AD} .{ }^{\mathrm{e} 20}$ While differences with the present results may be partly explained by the use of fluid vs imaging markers, which capture different aspects of tau pathology, ${ }^{\mathrm{e} 21}$ and midlife vs late life assessments, these results may also highlight different paths through which stress may affect brain health, including disease-independent pathways.

Resilience to stress and stress coping ability depends upon demographic factors such as age, sex, and education. ${ }^{\text {e22 }}$ In this study, stress coping ability was not associated with age or sex, as reported previously. Although some studies suggest differences in tau burden by sex, ${ }^{\text {e23 }}$ there was no interaction between sex and stress coping ability on tau. This question, however, needs further assessment in future studies using gender-sensitive tools. Aside from age and sex, lifestyle habits may be associated with stress and warrant further investigation in future studies. Further, future studies may also benefit from measuring stressful life events and social support, both important for stress resilience. Finally, unraveling the mechanism through which stress plays a role in $\mathrm{AD}$ clinical syndrome will be fundamental to designing successful interventions. If stress increases tau accumulation in aging, stress resilience could be an area for intervention earlier in life. In addition, stress may accelerate the $\mathrm{AD}$ biomarker cascade, in which case stress regulation could be a potential target for interventions in participants with $\mathrm{AD}$ pathologic change. On the other hand, if the stress response is altered early in the disease process, then stress assessment can be used for early detection and treatment. Future studies are warranted to assess the associations between stress resilience and tau in cognitively impaired participants.

The main limitation of the present study is that its crosssectional design does not allow assessing causality. The association between stress and tau needs further assessment using other measurements of stress such as cortisol levels. However, it is important to highlight that stress responses depend upon different variables including resilience resources and objective exposure to stressors that may influence stress coping ability and were not measured in the present study. Our results highlight that measuring the ability to cope or bounce back from stress may be clinically relevant and important to study stress in the context of aging.

Higher stress coping ability was associated with lower tau in aging and amyloid-positive $\mathrm{CU}$ older adults. Further studies are warranted to understand the role of stress in $\mathrm{AD}$ : stress may 
play a role in the development or promotion of tau pathology, or alternatively, lower stress coping ability may represent an early manifestation of accumulating tau pathology.

\section{Author contributions}

Dr. Arenaza-Urquijo: study concept and design, analysis and interpretation. Dr. Machulda: interpretation, critical revision of the manuscript for important content. Dr. Knopman: interpretation, critical revision of the manuscript for important intellectual content. Dr. Lowe: interpretation, critical revision of the manuscript for important intellectual content. Dr. Mielke: critical revision of the manuscript for important intellectual content. A.L. Reddy: acquisition of data and analysis. Dr Geda: critical revision of the manuscript for important intellectual content. Dr. Jack Jr: study design, interpretation, critical revision of the manuscript for important intellectual content. Dr. Petersen: study design, interpretation, critical revision of the manuscript for important intellectual content. Dr. Vemuri study concept and design, interpretation, critical revision of the manuscript for important intellectual content, study supervision.

\section{Study funding}

The authors thank the study participants and staff in the Mayo Clinic Study of Aging, Mayo Alzheimer's Disease Research Center, and Aging Dementia Imaging Research Laboratory at the Mayo Clinic; and Avid Radiopharmaceuticals, Inc., for their support in supplying AV-1451 precursor, chemistry production advice and oversight, and FDA regulatory crossfiling permission and documentation needed for this work. This work was supported by NIH grants R01 AG56366 (PI: P.V.), U01 AG06786 (PI: R.C.P.), R01 NS097495 (PI: P.V.), P50 AG16574/P1 (PI: P.V.), P50 AG16574 (PI: R.C.P.), R01 AG11378 (PI: C.R.J.), R01 AG041851 (PIs: C.R.J. and D.S.K.), and R01 AG55151 (PI: M.M. Mielke); the Gerald and Henrietta Rauenhorst Foundation grant; the Millis Family; the Alexander Family Alzheimer's Disease Research Professorship of the Mayo Foundation; Liston Award; Elsie and Marvin Dekelboum Family Foundation; Schuler Foundation; and Opus building NIH grant C06 RR018898, and was made and made possible by the Rochester Epidemiology Project (R01 AG034676). The funding sources were not involved in the manuscript review or approval.

\section{Disclosure}

E. Arenaza-Urquijo and S. Przybelski report no disclosures relevant to the manuscript. M. Machulda receives funding from the NIH. D. Knopman serves on a Data Safety Monitoring Board for the DIAN study, is an investigator in clinical trials sponsored by Biogen, Lilly Pharmaceuticals, and the University of Southern California, and receives research support from the NIH. V. Lowe consults for Bayer Schering Pharma, Piramal Life Sciences, and Merck Research and receives research support from GE Healthcare, Siemens Molecular Imaging, Avid Radiopharmaceuticals, and the NIH (National Institute on Aging [NIA], National Cancer Institute). M. Mielke served as a consultant to Eli Lilly and Lysosomal Therapeutics, Inc., and receives research support from the NIH (R01 AG49704, P50 AG44170, U01 AG06786, RF1 AG55151, U54 AG44170), Department of Defense (W81XWH-15-1), and unrestricted research grants from Biogen, Roche, and Lundbeck. A. Reddy reports no disclosures relevant to the manuscript. Y. Geda receives research support from the NIH (AG 057708) and an unrestricted grant from Roche. C. Jack Jr. receives NIH grants R37 AG011378 and R01 AG 041851 and the Alexander Family Alzheimer's Disease Research Professorship of the Mayo Clinic. C. Jack consults for Eli Lilly and serves on an independent data monitoring board for Roche but he receives no personal compensation from any commercial entity. $R$. Petersen receives grants from the NIA, NIA P50 AG016574, U01 AG006786, U01 AG024904, and the GHR Foundation, Alexander Family Foundation, and the Mayo Foundation for Medical Education and Research. Dr. Petersen consults for Roche, Inc., Merck, Inc., Genentech, Inc., Biogen, Inc., and GE Healthcare, and receives royalties from Oxford University Press for the publication of Mild Cognitive Impairment. P. Vemuri receives funding from the NIH (National Institute of Neurological Disorders and Stroke and NIA). Go to Neurology.org/ $\mathrm{N}$ for full disclosures.

\section{Publication history}

Received by Neurology March 7, 2019. Accepted in final form October 16, 2019.

\section{References}

1. Vemuri P. "Exceptional brain aging” without Alzheimer's disease: triggers, accelerators, and the net sum game. Alzheimers Res Ther 2018;10:53.

2. Arenaza-Urquijo EM, Vemuri P. Resistance vs resilience to Alzheimer disease: clarifying terminology for preclinical studies. Neurology 2018;90:695-703.

3. Carvalho DZ, St Louis EK, Knopman DS, et al. Association of excessive daytime sleepiness with longitudinal $\beta$-amyloid accumulation in elderly persons without dementia. JAMA Neurol 2018;75:672-680.

4. Mander BA, Winer JR, Jagust WJ, Walker MP. Sleep: a novel mechanistic pathway, biomarker, and treatment target in the pathology of Alzheimer's disease? Trends Neurosci 2016;39:552-566.

5. Brown BM, Peiffer JJ, Taddei K, et al. Physical activity and amyloid- $\beta$ plasma and brain levels: results from the Australian Imaging, Biomarkers and Lifestyle Study of Ageing. Mol Psychiatry 2013;18:875-881.

6. Okonkwo OC, Schultz SA, Oh JM, et al. Physical activity attenuates age-related biomarker alterations in preclinical AD. Neurology 2014;83:1753-1760.

7. Brown BM, Sohrabi HR, Taddei K, et al. Habitual exercise levels are associated with cerebral amyloid load in presymptomatic autosomal dominant Alzheimer's disease. Alzheimers Dement 2017;13:1197-1206.

8. Landau SM, Marks SM, Mormino EC, et al. Association of lifetime cognitive engagement and low $\beta$-amyloid deposition. Arch Neurol 2012;69:623-619.

9. Lo RY, Jagust WJ; Alzheimer's Disease Neuroimaging Initiative. Effect of cognitive reserve markers on Alzheimer pathologic progression. Alzheimer Dis Assoc Disord 2013;27:343-350.

10. Wirth M, Villeneuve S, La Joie R, Marks SM, Jagust WJ. Gene-environment interactions: lifetime cognitive activity, APOE genotype, and beta-amyloid burden. J Neurosci 2014;34:8612-8617.

11. Vemuri P, Lesnick TG, Przybelski SA, et al. Effect of intellectual enrichment on AD biomarker trajectories: longitudinal imaging study. Neurology 2016;86:1128-1135.

12. Arenaza-Urquijo EM, Bejanin A, Gonneaud J, et al. Association between educational attainment and amyloid deposition across the spectrum from normal cognition to dementia: neuroimaging evidence for protection and compensation. Neurobiol Aging 2017;59:72-79.

13. DeVos SL, Corjuc BT, Commins C, et al. Tau reduction in the presence of amyloid- $\beta$ prevents tau pathology and neuronal death in vivo. Brain 2018;141:2194-2212.

14. Li C, Götz J. Tau-based therapies in neurodegeneration: opportunities and challenges. Nat Rev Drug Discov 2017;16:863-883.

15. Brown BM, Rainey-Smith SR, Dore V, et al. Self-reported physical activity is associated with tau burden measured by positron emission tomography. J Alzheimers Dis 2018;63:1299-1305.

16. Almeida RP, Schultz SA, Austin BP, et al. Effect of cognitive reserve on age-related changes in cerebrospinal fluid biomarkers of Alzheimer disease. JAMA Neurol 2015; 72:699-706. 
17. Aguilera G. Regulation of pituitary ACTH secretion during chronic stress. Front Neuroendocrinol 1994;15:321-350.

18. Antoni FA. Hypothalamic control of adrenocorticotropin secretion: advances since the discovery of 41-residue corticotropin-releasing factor. Endocr Rev 1986;7: 351-378.

19. Sotiropoulos I, Catania C, Pinto LG, et al. Stress acts cumulatively to precipitate Alzheimer's disease-like tau pathology and cognitive deficits. J Neurosci 2011;31: $7840-7847$.

20. Green KN, Billings LM, Roozendaal B, McGaugh JL, LaFerla FM. Glucocorticoids increase amyloid-beta and tau pathology in a mouse model of Alzheimer's disease. J Neurosci 2006;26:9047-9056.

21. Jeong YH, Park CH, Yoo J, et al. Chronic stress accelerates learning and memory impairments and increases amyloid deposition in APPV717I-CT100 transgenic mice, an Alzheimer's disease model. FASEB J 2006;20:729-731.

22. Lopes S, Vaz-Silva J, Pinto V, et al. Tau protein is essential for stress-induced brain pathology. Proc Natl Acad Sci USA 2016;113:E3755-E3763.

23. Mejía S, Giraldo M, Pineda D, Ardila A, Lopera F. Nongenetic factors as modifiers of the age of onset of familial Alzheimer's disease. Int Psychogeriatr 2003;15: $337-349$.

24. Wilson RS, Evans DA, Bienias JL, Mendes de Leon CF, Schneider JA, Bennett DA. Proneness to psychological distress is associated with risk of Alzheimer's disease. Neurology 2003;61:1479-1485.

25. Johansson L, Guo X, Waern M, et al. Midlife psychological stress and risk of dementia: a 35-year longitudinal population study. Brain 2010;133:2217-2224.

26. Wang HX, Gustafson DR, Kivipelto M, et al. Education halves the risk of dementia due to apolipoprotein $\varepsilon 4$ allele: a collaborative study from the Swedish brain power initiative. Neurobiol Aging 2012;33:1007.e1-7.

27. Crowe M, Andel R, Pedersen NL, Gatz M. Do work-related stress and reactivity to stress predict dementia more than 30 years later? Alzheimer Dis Assoc Disord 2007; 21:205-209.

28. Lupien SJ, de Leon M, de Santi S, et al. Cortisol levels during human aging predict hippocampal atrophy and memory deficits. Nat Neurosci 1998;1:69-73.

29. Lupien SJ, Maheu F, Tu M, Fiocco A, Schramek TE. The effects of stress and stress hormones on human cognition: implications for the field of brain and cognition. Brain Cogn 2007;65:209-237.

30. Csernansky JG, Dong H, Fagan AF, et al. Plasma cortisol and progression of dementia in DAT subjects. Am J Psychiatry 2006;163:2164-2169.

31. Popp J, Wolfsgruber S, Heuser I, et al. Cerebrospinal fluid cortisol and clinical disease progression in MCI and dementia of Alzheimer's type. Neurobiol Aging 2015;36: 601-607.

32. Lehallier B, Essioux L, Gayan J, et al. Combined plasma and cerebrospinal fluid signature for the prediction of midterm progression from mild cognitive impairment to Alzheimer disease. JAMA Neurol 2016;73:203-212.

33. Ennis GE, An Y, Resnick SM, et al. Long-term cortisol measure predict Alzheimer disease risk. Neurology 2017;88:371-378.
34. Giubilei F, Patacchioli FR, Antonini G, et al. Altered circadian cortisol secretion in Alzheimer's disease: clinical and neuroradiological aspects. J Neurosci Res 2001;66: 262-265

35. Piertzak RH, Laws SM, Lim YY, et al. Plasma cortisol, brain amyloid- $\beta$, and cognitive decline in preclinical Alzheimer's disease: a 6-year prospective cohort study. Biol Psychiatry Cogn Neurosci Neuroimaging 2017;2:45-52.

36. Aisen PS, Davis KL, Berg JD, et al. A randomized controlled trial of prednisone in Alzheimer's disease: Alzheimer's Disease Cooperative Study. Neurology 2000;54: 588-593.

37. Pittenger C, Duman RS. Stress, depression, and neuroplasticity: a convergence of mechanisms. Neuropsychopharmacology 2008;33:88-109.

38. Charney DS, Manji HK. Life stress, genes, and depression: multiple pathways lead to increased risk and new opportunities for intervention. Sci STKE 2004;2004:re5.

39. Gaffey AE, Bergeman CS, Clark LA, Wirth MM. Aging and the HPA axis: stress and resilience in older adults. Neurosci Biobehav Rev 2016;68:928-945.

40. Fleshner M, Maier SF, Lyons DM, Raskind MA. The neurobiology of the stressresistant brain. Stress 2011;14:498-502.

41. Sampedro-Piquero P, Alvarez-Suarez P, Begega A. Coping with stress during aging: the importance of a resilient brain. Curr Neuropharmacol 2018;16:284-296.

42. Sood A, Prasad K, Schroeder D, Varkey P. Stress management and resilience training among Department of Medicine faculty: a pilot randomized clinical trial. J Gen Intern Med 2011;26:858-861.

43. Smith BW, Dalen J, Wiggins K, Tooley E, Christopher P, Bernard J. The brief resilience scale: assessing the ability to bounce back. Int J Behav Med 2008;15: 194-200.

44. Stranahan AM, Mattson MP. Selective vulnerability of neurons in layer II of the entorhinal cortex during aging and Alzheimer's disease. Neural Plast 2010;2010: 108190.

45. Rocca WA, Yawn BP, St Sauver JL, Grossardt BR, Melton LJ. History of the Rochester Epidemiology Project: half a century of medical records linkage in a US population. Mayo Clin Proc 2012;87:1202-1213.

46. Sauver JLS, Grossardt BR, Yawn BP, et al. Data resource profile: the Rochester Epidemiology Project (REP) medical records-linkage system. Int J Epidemiol 2012; 41:1614-1624.

47. Petersen RC, Roberts RO, Knopman DS, et al. Prevalence of mild cognitive impairment is higher in men: The Mayo Clinic Study of Aging. Neurology 2010;75: 889-897.

48. Beck AT, Steer RA, Brown GK. Beck Depression Inventory-II Manual. San Antonio Psychological Corporation; 1996.

49. Beck AT, Epstein N, Brown G, Steer RA. An inventory for measuring clinical anxiety: psychometric properties. J Consult Clin Psychol 1988;56:893-897.

50. Jack CR, Wiste HJ, Weigand SD, et al. Defining imaging biomarker cut points for brain aging and Alzheimer's disease. Alzheimers Dement 2017;13:205-216. Data available from Dryad (Additional References, References e1 to e23) doi.org/10. 5061/dryad.0zpc866t7 


\section{Neurology}

\section{Better stress coping associated with lower tau in amyloid-positive cognitively unimpaired older adults \\ Eider M. Arenaza-Urquijo, Scott A. Przybelski, Mary M. Machulda, et al.}

Neurology 2020;94;e1571-e1579 Published Online before print January 21, 2020

DOI 10.1212/WNL.0000000000008979

This information is current as of January 21, 2020

\section{Updated Information \&} Services

References

Citations

Subspecialty Collections

Permissions \& Licensing

Reprints including high resolution figures, can be found at: http://n.neurology.org/content/94/15/e1571.full

This article cites 49 articles, 12 of which you can access for free at: http://n.neurology.org/content/94/15/e1571.full\#ref-list-1

This article has been cited by 2 HighWire-hosted articles: http://n.neurology.org/content/94/15/e1571.full\#\#otherarticles

This article, along with others on similar topics, appears in the following collection(s):

\section{Alzheimer's disease}

http://n.neurology.org/cgi/collection/alzheimers_disease

PET

http://n.neurology.org/cgi/collection/pet

Information about reproducing this article in parts (figures,tables) or in its entirety can be found online at:

http://www.neurology.org/about/about_the_journal\#permissions

Information about ordering reprints can be found online:

http://n.neurology.org/subscribers/advertise

Neurology ${ }^{\circledR}$ is the official journal of the American Academy of Neurology. Published continuously since 1951 , it is now a weekly with 48 issues per year. Copyright Copyright (C) 2020 The Author(s). Published by Wolters Kluwer Health, Inc. on behalf of the American Academy of Neurology.. All rights reserved. Print ISSN: 0028-3878. Online ISSN: 1526-632X.

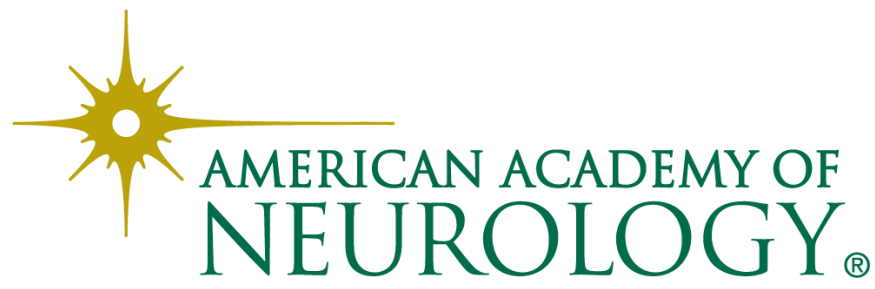

